\title{
Audiological Profiling and Rehabilitation Outcomes in a Child With Johanson-Blizzard Syndrome
}

\author{
Aiza Fatima Raza, Dilli Raj Paudel, and Kavassery Venkateswaran Nisha \\ Department of Audiology, All India Institute of Speech and Hearing (AllSH), Naimisham Campus, Mysore, India
}

\author{
Received July 26, 2021 \\ Revised August 20,2021 \\ Accepted September 6, 2021 \\ Address for correspondence \\ Kavassery Venkateswaran Nisha, \\ $\mathrm{PhD}$ \\ Department of Audiology, \\ All India Institute of Speech and \\ Hearing, Naimisham Campus, \\ Manasagangothri, \\ Mysore-570006, India \\ Tel +91-0821-2502581 \\ Fax +91-9035362891 \\ E-mailnnishakv@aiishmysore.in
}

Johanson Blizzard syndrome (JBS) is an autosomal recessive disorder that shows a multi-faceted impact on almost all body functions, including speech and hearing. This case presentation describes the comprehensive audiological and rehabilitative profile of an 8-year-old female child with JBS while correlating the test results to the physiological aspects of hearing. Case history revealed poor developmental motor skills, delayed speech and language development with hypothyroidism, and dysmorphic facial features including low bat ears, micrognathia, high arched palate, and hypoplasia of nasal alae. Conditioned pure-tone audiometric responses revealed profound hearing loss of cochlear origin in both ears, which was substantiated with bilateral Atype tympanogram in immittance evaluation. Otoacoustic emissions and auditory brain stem response were absent in both ears, consistent with the audiometric findings. Rehabilitation attempts with a cochlear implant and hearing aid in the opposite ears showed differential improvements, which were in harmony with the aided thresholds. The physiological basis for each finding and future implications are discussed.

J Audiol Otol 2022;26(3):160-165

\section{Introduction}

Described in 1971 for the first time by Johanson and Blizzard, Johanson Blizzard syndrome (JBS) is an autosomal recessive disorder caused by mutations to the ubiquitin protein ligase E3 component N-recognin 1 gene [1,2]. The predominance of JBS is more in populations with a high rate of consanguineous marriages [3]. No reports on the incidence and prevalence of JBS are available in the Indian scenario.

Detailed analyses of the symptoms in JBS show the multifaceted impact of the disorder on almost all the body systems. The associated abnormalities listed in order of prevalence in patients with JBS are pancreatic insufficiency (98\%), nasal alae hypoplasia (98\%), dental hypoplasia/agenesis (96\%), short stature (84\%), scalp defects (72\%), deafness (69\%), and mental retardation $(69 \%)$ [3,4]. In addition, JBS patients also exhibit cardiac and genitourinary abnormalities [5].

Most of the studies noted a hearing loss in JBS cases, al-

This is an Open Access article distributed under the terms of the Creative Commons Attribution Non-Commercial License (https://creativecommons.org/licenses/by-nc/4.0/) which permits unrestricted non-commercial use, distribution, and reproduction in any medium, provided the original work is properly cited. though there is scanty literature supporting thorough documentation of the same. Sensorineural hearing loss (SNHL) of various degrees has been reported in JBS $[2,6,7]$. Ramos, et al. [8] discussed the temporal bone computed tomography (CT) scan findings report that outer, middle ear, and inner ear structure in a JBS individual show atresia of external auditory canal, normal left middle ear but soft tissues in the right side, normal inner ears, and internal acoustic meatus; except for aeration loss of the mastoid antrum and cells.

There is scanty literature documenting the auditory profile of JBS syndrome and relating it to aural rehabilitation in children. The lack of detailed studies in JBS warrants the need to explore the audiological characteristics of such individuals, which can help audiologists design the best possible rehabilitation measures. To this extent, the present study is one of the first attempts to profile and describe the audiological outcomes of an 8-year-old girl with JBS.

\section{Case Report}

\section{Ethical consideration}

The manuscript adheres to the ethical standards according 
to the declaration of Helsinki. Ethical approval was obtained from All India Institute of Speech and Hearing (AIISH) Review Board (ref: AIISH/20-2021: AUD_09). The aims of the study were explained to the parent, and written informed consent was obtained from the parent of the child before the start of the study.

\section{Patient information}

An eight-year, four-month-old female child diagnosed with JBS syndrome reported to the institute on Feb 25, 2020, with a complaint of decreased hearing in both ears since childhood. She is the only child of the parents.

Consanguinity of third-degree was present in the family history for hearing loss. A detailed case history, including prenatal, perinatal, and postnatal history-related details, was obtained through a structured interview with the child's parent. The mother reported a prenatal history of miscarriage and drug consumption. Postnatal history included neonatal jaundice, neonatal intensive care unit stay of five days for the same. Poor developmental motor skills, delayed speech, and language development were some of the other associated problems. Current medical history included the diagnosis of hypothyroidism. An attempt was also made to profile craniofacial features observed in the child. Dysmorphic facial features with low bat ears, micrognathia, high arched palate, nasal alae hypoplasia were noted in the case history. Genetic testing to confirm JBS was done on May 23, 2012. Bilateral Mondini dysplasia was diagnosed in the MRI and CT scan reports. Reports of other prior evaluations were not available.

\section{Testing protocol and clinical findings}

The psychological assessment conducted on Feb 28, 2020, at the institute using the developmental screening test (DST) revealed the child's intelligence quotient as 84 , suggestive of low average intelligence ability and the developmental age of 7. The otorhinolaryngologist at the institute examined the ear on Feb 25, 2020, and opined a clear external auditory canal with an intact tympanic membrane and a visible cone of light in the left ear. Impacted wax was present in the right ear, which was removed before the audiological evaluation. Detailed audiological profiling was done to evaluate the outer, middle, and inner ear status, while speech and language profiling assessed the child's language age and speech deficits.

\section{Audiological profiling}

Detailed audiological profiling was done to evaluate the status of the outer, middle, and inner ear. The details of audiological evaluations, including otoscopic examination, pure tone audiometry (PTA), immittance audiometry, otoacoustic emissions (OAE), auditory brainstem response (ABR). The audiological tests in the test battery were conducted in a double-blind manner. All the tests were performed by the audiologists (certified by Rehabilitation Council of India), who were blind to the study's objectives. The authors only profiled the test results obtained from the audiological test battery. All tests were performed in the same week, whenever possible on successive days, and the comprehensive audiological testing required approximately four days for completion. The procedure flowchart is presented in Fig. 1.

\section{Immittance audiometry}

A calibrated immittance meter, GSI Tympstar pro (GrasonStadler, Eden Prairie, MN, USA), was used to analyze the child's middle ear status. Tympanometry was performed bilaterally, using a $226 \mathrm{~Hz}$ probe tone. Acoustic reflex thresholds were measured at octave frequencies from 500, 1,000, 2,000, and 4,000 Hz ipsilateral and contralateral in both ears. A bilateral A-type tympanogram with absent reflexes and -9 daPa of tympanometric peak pressure, 0.91 and $0.86 \mathrm{mmho}$ of static admittance, and ear canal volume of $0.92 \mathrm{~cm}^{3}$ and $1.14 \mathrm{~cm}^{3}$ in the right and left ear, respectively, were reported. These findings are suggestive of no indication of middle ear pathology in both ears.

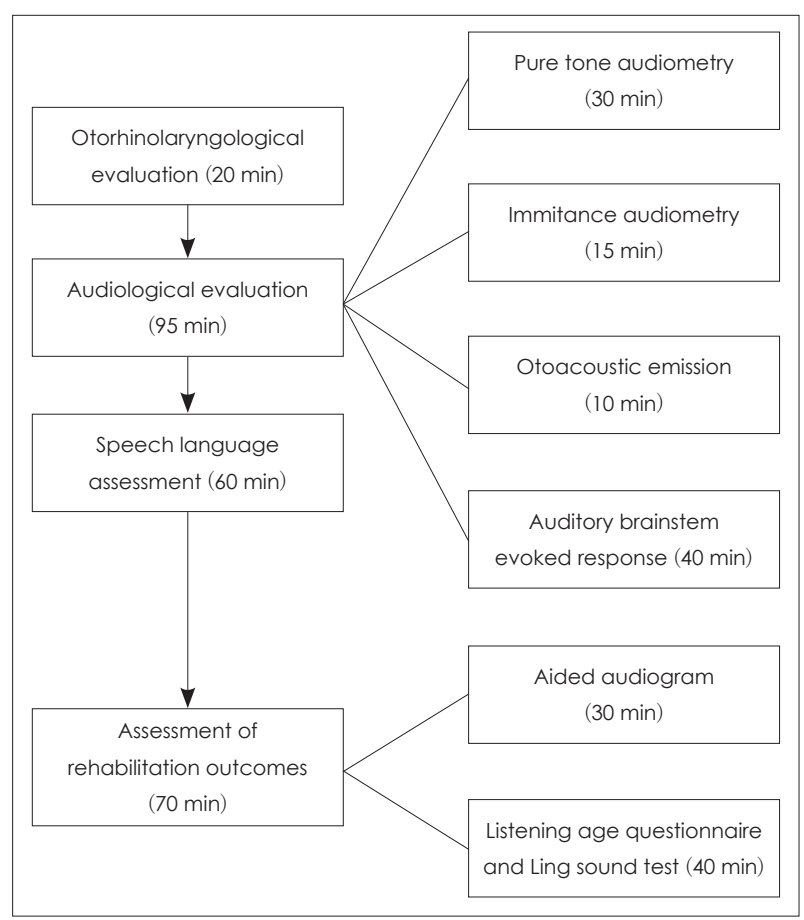

Fig. 1. Flowchart of audiological evaluation, speech-language assessment, and rehabilitation outcome measures. The time taken for each test is given in the parenthesis. 
Pure tone and speech audiometry

The pure tone and speech audiometry were performed in an acoustically treated room, which strict adherence to ambient condition standard prescribed by ANSI S3.1-1999. A calibrated dual-channel clinical audiometer, GSI Audiostar Pro (Grason-Stadler), was used to estimate the pure tone threshold and speech detection threshold. TDH-49 supra-aural earphones housed in MX 41/AR cushions were used to estimate air-conduction thresholds, while Radioear B-71 bone vibrator (RadioEar New Eagle, PA, USA) was used for bone conduction test. The thresholds were tracked at octave frequencies from $250 \mathrm{~Hz}$ to $8,000 \mathrm{~Hz}$ for air conduction and $250 \mathrm{~Hz}$ to 4,000 $\mathrm{Hz}$ for bone conduction testing. A provisional diagnosis of bilateral profound hearing loss was established in PTA, with the pure tone average being $>90 \mathrm{~dB}$ HL in both ears. The air and bone conduction thresholds across octave frequencies are shown in Fig. 2.

Distortion product otoacoustic emissions

Distortion product otoacoustic emissions (DPOAEs) were absent bilaterally across all the frequencies indicative of outer hair cell dysfunction in both the ears. The frequency band tested was from $1,000 \mathrm{~Hz}$ to $6,000 \mathrm{~Hz}$, with the ratio of F2: $\mathrm{F} 1=1.2: 1$. The stimulus intensity was maintained constant with level of stimulus 1 (L1) $=65 \mathrm{~dB}$ SPL \& level of stimulus $2(\mathrm{~L} 2)=55 \mathrm{~dB}$ SPL. A signal-to-noise ratio of at least $6 \mathrm{~dB}$ with a reproducibility score of at least $70 \%$ for at least 3 out of 6 frequency tested is considered for the presence of DPOAEs.

Auditory brainstem response (ABR)

Evoked auditory brainstem response (ABR) was carried out to check the integrity of the auditory system with Natus Biologic Navigator pro (Natus Medical Incorporated, San Carlos, CA, USA) Auditory Evoked Potentials (AEP) system using Eartone 3A insert phones (Etymotic Research, Elk Grove Village, IL, USA; electrode impedance $<5 \mathrm{kOhms}$ ). ABR was obtained in two paradigms: click and tone burst (TB) evoked ABRs. The first recording of ABR was done using click stimuli at a starting intensity at $90 \mathrm{dBnHL}$ with $11.1 / \mathrm{sec}$ in rarefaction polarity. This was followed by recording frequency-specific ABR using $500 \mathrm{~Hz}$ TB stimuli at $80 \mathrm{dBnHL}$ in alternating polarity with a stimulus rate of $11.1 / \mathrm{sec}$. Both the acquisition modalities had a high pass filter setting of $100 \mathrm{~Hz}$ and a low pass filter setting of 1,500 Hz. A notch filter at $60 \mathrm{~Hz}$ was kept on to prevent contamination from electrical artifacts. A time window of $10 \mathrm{~ms}$ was used for click-evoked ABR, and $15 \mathrm{~ms}$ for TB evoked ABR. A minimum of two traces was recorded for each paradigm to account for the replicability. Wave I, III, and $\mathrm{V}$ were tracked, and their absolute latencies were determined. Wave V could not be traced in both click and TB (500 $\mathrm{Hz}$ ) ABR bilaterally, indicating severe to profound hearing loss in both ears. The ABR waveforms of both ears are shown in Fig. 3.

\section{Speech language assessment}

Speech and language assessment was carried out using Scales of Early Communication Skills (SECS) [9] for hearing impaired children. Examination of oral peripheral mechanism, language development, social maturity, emotional stability, misarticulations was also carried out. A provisional diagnosis of spoken language disorder secondary to JBS was established. On SECS for the hearing impaired, receptive language

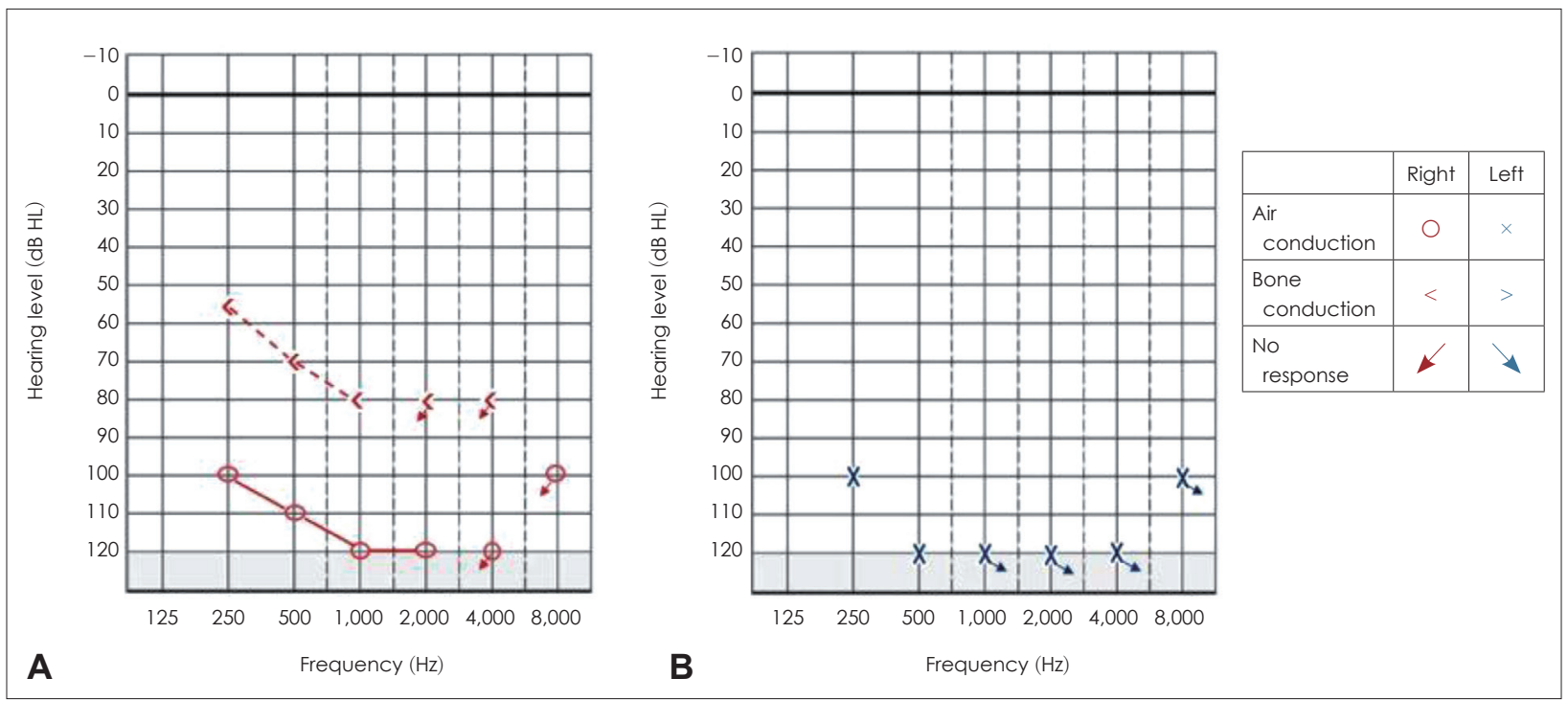

Fig. 2. Auditory thresholds corresponding to right ear (A) and left ear (B) in the test of pure tone audiometry. 


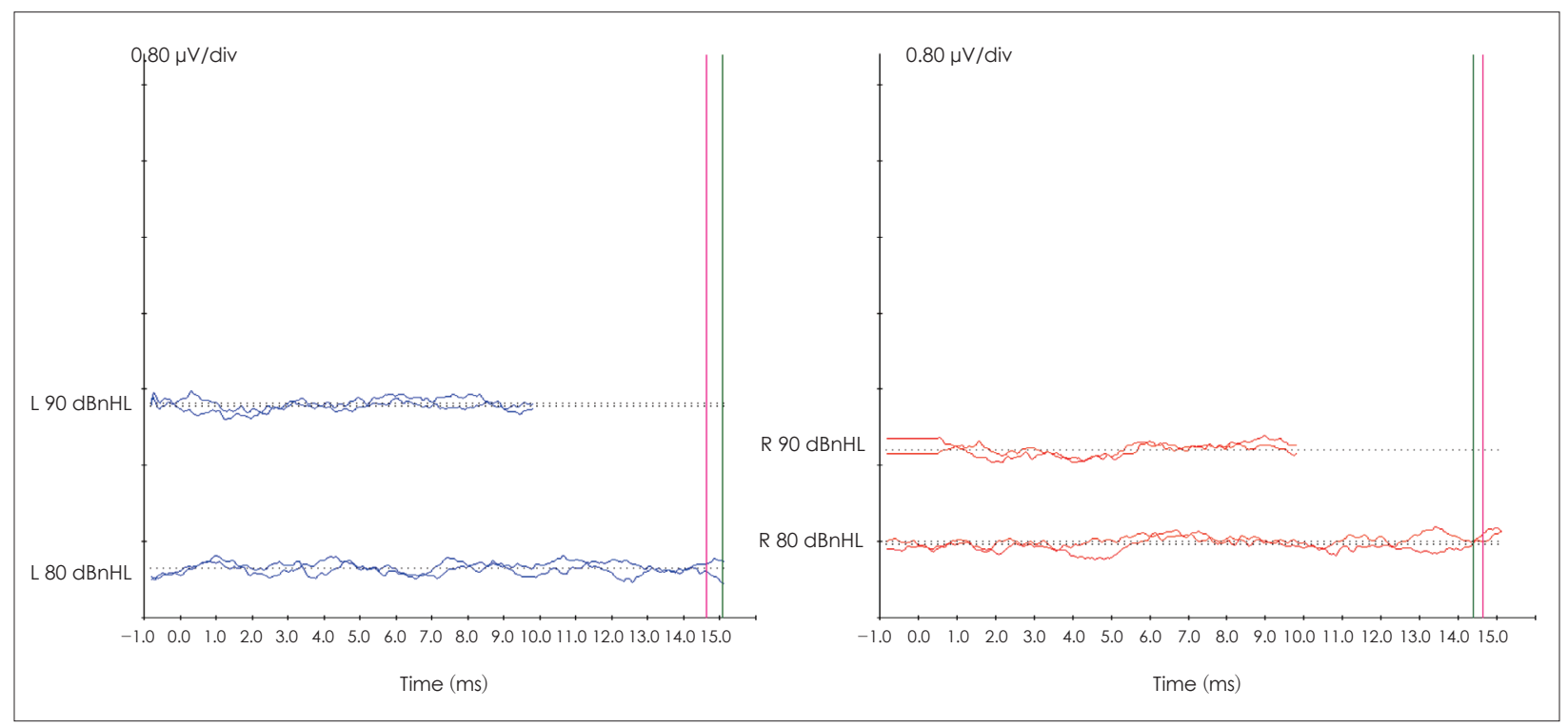

Fig. 3. Click and tone burst auditory brainstem response waveforms of left ear in blue and right ear in red.

age and expressive language age were less than 2.0 to 2.11 years. Oral peripheral mechanism examination revealed that both structure and function of oral structures were affected. Structural abnormality noticed were absence of permanent teeth, micrognathia and high arched palate. Receptive language consisted of reception of family members, fruits (apple banana, grapes, chikoo, oranges), vegetables (tomato, onion), animals (cow, dog, cat, horse) and common objects (pen, cap, chair, pant, mobile, table, plate). Expressive vocabulary consists of above mentioned family members, fruits, vegetables, common objects but with poor clarity. Child uses iconic gestures along with speech utterances. Misarticulations were present which further compromised speech intelligibility. Child used to indulge in solo play and group play and makes good eye contact.

\section{Rehabilitative outcomes}

The child had undergone cochlear implant (CI) surgery in the left ear on May 17, 2018. The child used an Advanced Bionics Naida CI Q70 cochlear implant (Sivantos Group, Piscataway, NJ, USA) in the left ear and a Siemens Reflex DSP hearing aid (Advanced Bionics, Los Angeles, CA, USA) in the right ear.

\section{Aided audiogram}

Unaided thresholds were determined in the free-field at 250, 500, 1,000, 2,000, and 4,000 Hz using frequency-specific warble tones. The unaided and aided free-field thresholds evaluated for pure tones with the hearing aid in the right ear and a CI in the left ear are shown in Fig. 4. A close visual inspection of Fig. 4 shows that the aided thresholds for the left

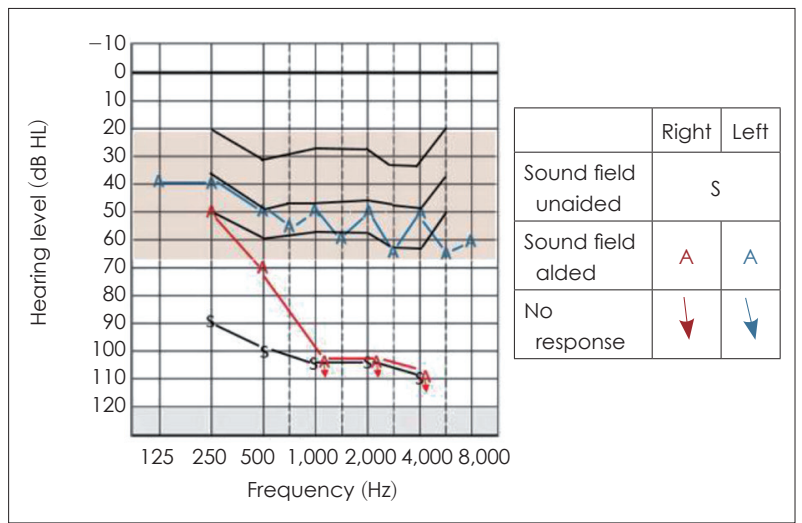

Fig. 4. Unaided sound field response denoted by "s". Aided right ear response with hearing aid " $A$ " in red and aided left ear response with cochlear implant " $A$ " in blue.

ear that received a CI were within the speech banana region, reflecting the benefit derived from $\mathrm{CI}$. The pure tone average was $>90 \mathrm{~dB}$ HL in the right ear and $50 \mathrm{~dB} \mathrm{HL}$ in the left ear.

Aided awareness and identification of Ling's six sounds were severely affected in the right ear, as shown in Tables 1 and 2, respectively. On the other hand, the awareness of Ling's six sounds was present in the left ear, but identification was present only for the /a/ sound. Closed set speech identification scores were 0 and 12 out of 25 in the right and left ear, respectively.

\section{Listening age questionnaire and Ling sound test}

The listening age of the child was established as 4-6 months using the listening age checklist [10]. Ling six sounds /a/, /i/, $/ \mathrm{u} /, / \mathrm{s} /, / \mathrm{J} /$, and $/ \mathrm{m} /$ were used to test awareness and identification of sounds in each sessions. Awareness of soft to moder- 
Table 1. Aided speech awareness threshold of Ling six sounds

\begin{tabular}{|c|c|c|c|c|c|c|}
\hline \multirow{2}{*}{ Ear } & \multicolumn{6}{|c|}{ Speech sound awareness levels ( $\mathrm{dB} \mathrm{HL}$ ) } \\
\hline & /a/ & /i/ & $/ \mathrm{u}$ & $\mid s /$ & $\mid \mathrm{S} /$ & $/ \mathrm{m} /$ \\
\hline Right ear with $\mathrm{HA}$ & NR at 80 & NR at 80 & NR at 80 & NR at 80 & NR at 80 & NR at 80 \\
\hline Left ear with $\mathrm{Cl}$ & 45 & 55 & 50 & 60 & 65 & 65 \\
\hline
\end{tabular}

$\mathrm{HA}$, hearing aid; $\mathrm{Cl}$, cochlear implant; $\mathrm{NR}$, no response

Table 2. Aided identification of Ling six sounds and closed set SIS

\begin{tabular}{|c|c|c|c|c|c|c|c|}
\hline \multirow{2}{*}{ Ear } & \multicolumn{6}{|c|}{ Aided Ling sound identification at $40 \mathrm{~dB} \mathrm{HL}$} & \multirow{2}{*}{ Aided SIS } \\
\hline & /a/ & /i/ & $/ \mathrm{u} /$ & $\mid s /$ & $/ 2 /$ & $/ \mathrm{m} /$ & \\
\hline Right ear with $\mathrm{HA}$ & NR & NR & NR & NR & NR & NR & $0 / 25$ \\
\hline Left ear with $\mathrm{Cl}$ & $\sqrt{ }$ & NR & NR & NR & NR & NR & $12 / 25$ \\
\hline
\end{tabular}

$(\sqrt{ })$ denotes the identification of ling sounds, respectively. HA, hearing aid; $\mathrm{Cl}$, cochlear implant; SIS, speech identification score; NR, no response

ate environmental sounds was present. The child could respond to name calls and environmental sounds and could discriminate between contrasting syllables. The therapy goals undertaken for the first five sessions were to improve awareness of danger sounds/warning signals such as vehicle horns so that child could associate meaning with it and become alert in the presence of such sounds and to improve discrimination of minimal pair contrasts such as baba, papa, mama. There was no change in the listening age after five sessions, after which therapy had to be discontinued due to nationwide lockdown. The parent was counselled about the home training activities and was also recommended for online therapy. Home training also included activities related to auditory awareness and discrimination. The parent was asked to report for a follow-up visit after six months.

\section{Discussion}

The present study findings highlight the need for a systematic and thorough auditory, speech and language profiling in children with JBS, apart from analyzing their anthropomorphic features. The findings of the study are the first of its kind to use the audiological test battery and report those systematically.

One of the constant signs needed to make a diagnosis of JBS includes facial dysmorphism. Dysmorphic facial features with low bat ears, micrognathia, high arched palate, nasal alae hypoplasia, absence of permanent teeth were observed in the case reported in the current study. These abnormalities observed in oral peripheral structures of speech mechanism in terms of its structure, in turn, could have a bearing on speech production function and could compound speech and language deficits (also secondary to poor audition), as evident in the case report. Several studies have reported that severe mental retardation can be present in the JBS population $[1,11]$. The child in the present study had low average intelligence on a DST. The audiological test battery (otoscopy, immittance evaluation, PTA, speech audiometry, aided audiogram, and Ling sound test) showed that deficits in JBS also manifest themselves in the form of hearing loss. The results of the visual examination of the external ear indicated no structural abnormalities of the pinna, ear canal, and tympanic membrane in the present case. In complimentary to the otoscopic findings of no structural abnormalities of external ear and canal, immittance evaluation revealed 'A' type tympanogram in both ears, which rules out the possibility of any subtle changes in the external ear or the middle ear (as indicated in the otoscopy). The combined results from otoscopy and immittance evaluations in our present case hint at internal ear dysfunction.

Results from PTA indicate bilateral symmetric profound hearing loss. These findings correspond to the findings of Rosanowoski, et al. [12] and Ramos, et al. [8], who pointed out deafness as one of the phenotypes of JBS. The severity of hearing loss can be idiosyncratic with the congenital abnormalities of the cochlea and inner ear structures. The current study's findings are in consensus with numerous syndromic hearing loss cases with inner ear involvement. Craniofacial syndromes involve hearing loss ranging from mild to moderate conductive to severe to profound SNHL. An examination of the oncogenicity shows that the inner ear (otic capsule), nasal alae, and mandible are ectodermal in origin [13]. Thus the facial features (aplastic nasal alae and micrognathia) along with the presence of severe to profound SNHL can be ontogenically related manifestations of ectodermal dysplasia.

The utility of amplification devices such as hearing aid, like the one used in the current study, is advocated to manage speech and language manifestations stemming from the disorder in mild to severe degrees of SNHL syndromic cases $[12,14]$. On the other hand, CIs are proven to be efficacious in treating syn- 
dromic severe to profound hearing loss cases [15]. However, in the present case, the prognosis with auditory rehabilitation measures was fairly low. Despite using these hearing amplification devices, the rehabilitative outcomes in the present case were not satisfactory due to the presence of bilateral Mondini dysplasia and late surgical intervention at the age of 7 . In addition, the below-average intellectual ability could have also compounded the aural rehabilitation outcomes seen in the present case. Although the child has passed critical age, functional learning can still take place. Lastly, the role of all allied professionals is also warranted to treat related domains of the disorder for the holistic development of the child.

In conclusion, the findings of the current study are first of its kind to use the audiological test battery and report the findings systematically. The test battery shows compromised auditory function, especially that of the inner ear as evident from the normal findings of otoscopic and immitance examination. From the present case report, it is also clear that hearing loss is a common feature of JBS, although it is scantly reported in the literature. CIs are proven to be effective in treating syndromic severe to profound hearing losses cases and should be involved in the management approach. The findings of this study also indicate the need for allied professional involvement to effectively screen, counsel, and successfully rehabilitate the children with JBS within the critical age itself, which can expedite both the rehabilitation and the audiological outcomes. Future studies are needed to profile the auditory, speech, and language longitudinal outcomes of the rehabilitation procedures in children with JBS. There is a need to report the importance of other demographic details such as parental education, motivation of the parent, socio-economic status, familial involvement, and support in therapy to quantify these rehabilitative outcomes effectively.

\section{Acknowledgments}

We would like to thank the Director and HOD, Audiology (All India Institute of Speech and Hearing, Mysuru, affiliated to the university of Mysuru) for permitting us to carry out the study. The authors like to thank all the participant and parent of the participant for their consent and cooperation during the data collection. This work did not receive any funding or grants.

\section{Conflicts of interest}

The authors have no financial conflicts of interest.

\section{Author Contributions}

Conceptualization: all authors. Data curation: Aiza Fatima Raza, Dilli Raj Paudel. Formal analysis: Kavassery Venkateswaran Nisha. Investigation: all authors. Methodology: Kavassery Venkateswaran
Nisha. Project administration: Aiza Fatima Raza, Dilli Raj Paudel. Resources: Aiza Fatima Raza, Dilli Raj Paudel. Software: Aiza Fatima Raza, Dilli Raj Paudel. Supervision: Kavassery Venkateswaran Nisha. Visualization: Aiza Fatima Raza, Dilli Raj Paudel. Writingoriginal draft: all authors. Writing — review \& editing: Kavassery Venkateswaran Nisha.

\section{ORCID iDs}

Aiza Fatima Raza https://orcid.org/0000-0003-0364-2677

Dilli Raj Paudel https://orcid.org/0000-0001-5968-8995

Kavassery Venkateswaran Nisha

https://orcid.org/0000-0003-0788-1800

\section{REFERENCES}

1) Zenker M, Mayerle J, Lerch MM, Tagariello A, Zerres K, Durie PR, et al. Deficiency of UBR1, a ubiquitin ligase of the N-end rule pathway, causes pancreatic dysfunction, malformations and mental retardation (Johanson-Blizzard syndrome). Nat Genet 2005;37:134550.

2) Atik T, Karakoyun M, Sukalo M, Zenker M, Ozkinay F, Aydoğdu S. Two novel UBR1 gene mutations in a patient with Johanson Blizzard syndrome: a mild phenotype without mental retardation. Gene 2015;570:153-5.

3) Almashraki N, Abdulnabee MZ, Sukalo M, Alrajoudi A, Sharafadeen I, Zenke M. Johanson-Blizzard syndrome. World J Gastroenterol 2011;17:4247-50

4) Alkhouri N, Kaplan B, Kay M, Shealy A, Crowe C, Bauhuber S, et al. Johanson-Blizzard syndrome with mild phenotypic features confirmed by UBR1 gene testing. World J Gastroenterol 2008;14:6863-6.

5) Johanson A, Blizzard R. A syndrome of congenital aplasia of the alae nasi, deafness, hypothyroidism, dwarfism, absent permanent teeth, and malabsorption. J Pediatr 1971;79:982-7.

6) Baleva LS, Sipyagina AE, Zenker M, Kagan JM, Danilycheva LI, Sucalo M. Occurrence of the rare syndrome of Johanson-Blizzard in a child-longitudinal observations. J Dev Disabil 2015;21:119-24.

7) Jafari SA, Moghaddar R, Bahadoram M, Kianifar HR, Tosi MB. A case with complete pancreatic aplasia suggestive of Johanson-Blizzard syndrome. J Clin Diagnostic Res 2016;10:SD01-3.

8) Ramos S, Ramos H, Ramos R, Piexito C, Ramos B. Johanson-Blizzard syndrome. Braz J Otorhinolaryngol 2010;76:794.

9) Moog JS, Geers AE. Scales of early communication skills for hearing-impaired children. Saint Louis, MO: Central Institute for the Deaf; 1975.

10) Loaney M, Richards L, Romanik S. Listen, learn and talk. Lane Cove West, Australia: Cochlear Ltd;2005.

11) Hurst JA, Baraitser M. Johanson-Blizzard syndrome. J Med Genet 1989;26:45-8.

12) Rosanowski F, Hoppe U, Hies T, Eysholdt U. Johanson-Blizzard syndrome. A complex dysplasia syndrome with aplasia of the nasal alae and inner ear deafness. HNO 1998;46:876-8.German.

13) Northern JL, Downs MP. Hearing in children. 5th ed. PA: Lippincott Williams \& Wilkins;2002.

14) Aubrey S, Crooks B, Rashid M. Pancytopenia from severe cobalamin (vitamin B12) deficiency in Johanson-Blizzard syndrome. Eur J Clin Nutr 2013;67:1118.

15) Holcomb MA, Rizk HG, Morris NS, Meyer TA. Bilateral cochlear implantation in a child with Johanson Blizzard syndrome. Int J Pediatr Otorhinolaryngol 2017;95:69-71. 